\title{
Linear Response Theory and the KMS Condition
}

\author{
J. Naudts \\ University of Antwerpen, Antwerpen, Belgium \\ A. Verbeure and R. Weder ${ }^{\star}$ \\ University of Leuven, Heverlee, Belgium $\star \star$
}

Received November 15, 1974

\begin{abstract}
The response, relaxation and correlation functions are defined for any vector state $\omega$ of a von Neumann algebra $\mathfrak{M}$, acting on a Hilbert space $\mathscr{H}$, satisfying the KMS-condition. An operator representation of these functions is given on a particular Hilbert space $\tilde{\mathscr{H}}$.

With this technique we prove the existence of the static admittance and the relaxation function. Finally we generalize the fluctuation-dissipation theorem and other relations between the above mentionned functions to infinite systems.
\end{abstract}

\section{Introduction}

In conventional statistical mechanics an equilibrium state of a finite system is given by a Gibbs state. It is well known that the states of infinite continuous systems are no longer of this type. It has been suggested and now widely accepted that an equilibrium state of an infinite system should be described by a state satisfying the KMS-condition [1]. This is also the point of view of this paper.

The problem of non-equilibrium statistical mechanics is to explain the occurrence of an equilibrium state. This problem can be tackled in different ways. There is a direction where people study the problem by placing the system in a larger one. This leads to the study of open systems, where topics like the master equation are widely studied $[2,3]$, some aspects of the theory have recently been made rigorous [4-6]. Also a lot of rigorous work has been done on models, such as harmonic oscillators and lasers (see e.g. [7]). Another way of studying the problem is to consider small perturbations of the system and to wait for the behaviour after a long time (see e.g. $[8,9]$ ). Linear response theory must be situated in this direction and the principal purpose of the present paper is to prove and generalize to infinite systems rigorously some aspects of linear response theory, as introduced by Kubo [10] and Mori [11].

In Section II we introduce a new scalar product on the set of observables and define a new Hilbert space $\tilde{\mathscr{H}}$, and construct explicity a unitary operator from $\tilde{\mathscr{H}}$ to the KMS-Hilbert space. We prove that it is equivalent with the scalar product of the Kubo-Mori theory, and we give some other characterizations of this scalar product.

$\star$ Onderzoeker IIKW, Belgium.

$\star \star$ Postal address: Institute for Theoretical Physics, Celestijnenlaan 200 D, B-3030 Heverlee, Belgium. 
In Section III we define the correlation, response, relaxation and admittance functions of linear response theory. We give an operator representation of this functions on $\tilde{\mathscr{H}}$. This technique enables us to prove in an easy way the existence of the static admittance and the relaxation function for all pairs of observables, and also to give their explicit forms. Other results which we want to mention are the proof of the fluctuation-dissipation theorem for infinite-system equilibrium states, and the proof of other relations between the above-mentioned functions. Finally we mention the proof of the Bogoliubov inequality for infinite system equilibrium states, as a straightforward application of the techniques, which are used.

The formalism developed here, for linear response theory is applied in a subsequent paper [19], where the importance of this theory is indicated in the study of cluster properties.

\section{The KMS-Condition and the Hilbert Space $\tilde{\mathscr{H}}$}

Let $\mathfrak{M}$ be a von Neumann algebra on a Hilbert space $\mathscr{H}$; let $t \rightarrow U_{t}$ be a strongly continuous map from the real numbers $\mathbb{R}$ into the group of unitaries on $\mathscr{H}$, then there exists a self-adjoint operator $H$ on $\mathscr{H}$ such that $U_{t}=\operatorname{expit} H$ and let $x_{t}=U_{t} x U_{t}^{*}$; furthermore let $\omega$ be any vector state on $\mathfrak{M}$ i.e. $\omega(x)=(\Omega, x \Omega)$ for all $x \in \mathfrak{M}$ with $\Omega$ a cyclic element of $\mathscr{H}$; the state $\omega$ is an equilibrium state if it satisfies the following definition.

Definition II.1. The state $\omega$ on $\mathfrak{M}$ satisfies the KMS-condition at inverse temperature $\beta=1 / k T$ if for any pair $(x, y)$ of $\mathfrak{M}$, there exists a complex function $F_{x, y}(z)$, defined, bounded and continuous on the strip $-\beta \leqq \operatorname{Im} z \leqq 0$, and analytic inside, with boundary values:

$$
\begin{aligned}
& F_{x, y}(t)=\omega\left(x_{t} y\right), \\
& F_{x y}(t-i \beta)=\omega\left(y x_{t}\right) .
\end{aligned}
$$

Let us first quote some consequences of the KMS-condition [12]. If $\omega$ satisfies the properties of Definition II.1, then the vector $\Omega$ is also separating for $\mathfrak{M}$, furthermore $\Omega$ is $U_{t}$-invariant i.e. $U_{t} \Omega=\Omega$ for all $t \in \mathbb{R}$.

There exists an operator $\Delta=\exp (-\beta H)$, given by

$$
\Delta=\exp (-\beta H)=\int_{-\infty}^{\infty} e^{-\beta \lambda} E(d \lambda)
$$

where $E(\lambda)$ is the spectral family of $H[12$, p. 69].

This operator is called the modular operator. It can be written as $\Delta=F S$ where $S$ is the closure of the conjugate linear operator, mapping $x \Omega$ into $x^{*} \Omega$ with domain $\mathfrak{M} \Omega$ and $F$ the adjoint of $S$. Let $S=J \Delta^{1 / 2}$ be the polar decomposition of $S$ then $J$ is an anti-unitary operator and $F=\Delta^{-1 / 2} J$; furthermore $J \Delta^{i t}=\Delta^{i t} J$ for all $t \in \mathbb{R}$ and $U_{t}=\Delta^{i t}, J \Omega=\Omega, \Delta \Omega=\Omega$.

Finally we quote the following property which is used afterward:

$$
\begin{aligned}
& F_{x, y}(\beta z)=\left(x^{*} \Omega, \Delta^{i z} y \Omega\right) \quad \text { if } \quad 0 \geqq \operatorname{Im} z \geqq-1 / 2, \\
& F_{x, y}(\beta z)=\left(y^{*} \Omega, \Delta^{1-i z} x \Omega\right) \quad \text { if } \quad-1 / 2 \geqq \operatorname{Im} z \geqq-1 .
\end{aligned}
$$


In the following we will always assume that $\omega$ is a KMS-state and for notational convenience we put $\beta=1$.

Define the unbounded operator $T$ by

$T=\int_{-\infty}^{\infty}\left(\frac{1-e^{-\lambda}}{\lambda}\right)^{1 / 2} E(d \lambda)$.

As is easily checked $T$ is a positive, self-adjoint operator, and as, for $\lambda \rightarrow-\infty$

$$
\left(\frac{1-e^{-\lambda}}{\lambda}\right)^{1 / 2} \sim\left(\frac{-e^{-\lambda}}{-\lambda}\right)^{1 / 2}<\left(e^{-\lambda}\right)^{1 / 2}
$$

the domain $\mathscr{D}\left(\Delta^{1 / 2}\right)$ of $\Delta^{1 / 2}$ is contained in the domain $\mathscr{D}(T)$ of $T$ and $\mathfrak{M} \Omega \subset \mathscr{D}(T)$.

We define the following sesquilinear form on $\mathfrak{M}$ :

$$
(x, y)_{\sim}=(T x \Omega, T y \Omega) \quad x, y \in \mathfrak{M} .
$$

Lemma II.2. The sesquilinear form $(\cdot, \cdot)$ on $\mathfrak{M}$ is non degenerated.

Proof. Let $(x, x)_{\sim}=0$ then $T x \Omega=0$. If we prove that zero is not an eigenvalue of $T$, using the fact that $\Omega$ is separating for $\mathfrak{M}$, it follows that $x=0$.

Let $W(\lambda)$ be the spectral family associated with the operator $(-T)$. Then [18].

$$
W(S)=E\left(\psi^{-1}(S)\right),
$$

for any Borel subsets of the real line, where $\psi^{-1}(S)$ denotes the inverse image of $S$ under the function $\psi(\lambda)=-\left(\frac{1-e^{-\lambda}}{\lambda}\right)^{1 / 2}$.

In particular $W((-\varepsilon, 0])=E((b, \infty])$, where $\varepsilon>0$, and $b$ is the inverse image under $\psi$ of $-\varepsilon$. Clearly

$$
\lim _{\varepsilon \rightarrow 0} b(\varepsilon)=\infty,
$$

then

$$
\lim _{\varepsilon \rightarrow 0} W((-\varepsilon, 0])=\lim _{b \rightarrow \infty} E((b, \infty])=0 .
$$

So that, 0 is not an eigenvalue of $T$, because it is not an eigenvalue of $(-T)$.

It follows from this Lemma that the closure of $\mathfrak{M}$ with respect to the sesquilinear form $(\cdot, \cdot)$ is a Hilbert space, which we denote by $\tilde{\mathscr{H}}$.

Following Ref. [12] let $\mathscr{E}$ be the linear space spanned by elements $f * g$, where $f$ and $g$ are continuous functions of a real variable with compact support, and where

$$
(f * g)(t)=\int_{-\infty}^{+\infty} f(s) g(t-s) d s
$$

is the convolution product.

Denote by $\mathfrak{B}$ the subalgebra of $\mathfrak{M}$ generated by the set

$$
\begin{aligned}
& \{f(H) x \mid f \in \mathscr{E}, x \in \mathfrak{M}\}, \\
& f(H)=\int_{-\infty}^{\infty} f(\lambda) E(d \lambda) \quad[12, \text { p. 67]. }
\end{aligned}
$$


$\mathfrak{B}$ is called the set of analytic elements of $\mathfrak{M}$ and has the following properties

(i) $\mathfrak{B}$ is invariant under $\Delta^{\alpha}, \alpha \in \mathbb{C}$ (complex numbers),

(ii) $\mathfrak{B}$ is dense in the Hilbert space $\mathscr{D}\left(\Delta^{\alpha}\right), \alpha \in \mathbb{C}$.

Theorem II.3. The operator $U$ from $\tilde{\mathscr{H}}$ into $\mathscr{H}$, defined by $U x=T x \Omega$ for all $x \in \mathfrak{M}$, extends to an unitary operator from $\tilde{\mathscr{H}}$ to $\mathscr{H}$.

Proof. From the relation

$$
\|U x\|=\|T x \Omega\|=\|x\|_{\sim} \quad \text { for } \quad x \in \mathfrak{M}
$$

it follows that $U$ has a continuous isometric extension from $\tilde{\mathscr{H}}$ into $\mathscr{H}$. We denote this extension by the same symbol. If we prove that the range, $R(T)$, of $T$ is dense in $\mathscr{H}$ and that $R(T) \subseteq R(U)$ where $R(U)$ is the range of $U$, the theorem is proved. First we prove that the range, $R(T)$, of $T$ is dense in $\mathscr{H}$.

As $T$ is selfadjoint

$$
(R(T))^{\perp}=N(T) \quad[14, \text { p. } 267],
$$

but $N(T)=\{0\}$; hence $(R(T))^{\perp}=\{0\}$, i.e. $R(T)$ is dense in $\mathscr{H}$. Now we prove that $R(T) \subseteq R(U)$ : let $v \in R(T)$, then $v=T u$ for some $u \in \mathscr{D}(T)$, as $\mathfrak{B} \Omega$ is a core for $f(H)$, where $f(\lambda)$ is a continuous function of a real parameter $[12$, p. 67], there exists a sequence $\left\{x_{n}\right\}$ belonging to $\mathfrak{B}$ such that $\lim _{n} x_{n} \Omega=u$ and $\lim _{n} T x_{n} \Omega=v$.

Hence $\left\|T x_{n} \Omega-T x_{m} \Omega\right\|=\left\|x_{n}-x_{m}\right\|_{\sim}$ tends to zero for $n$ and $m$ large enough. As $\tilde{\mathscr{H}}$ is closed there exists an element $x \in \tilde{\mathscr{H}}$ such that $x=\lim _{n} x_{n}$ and $v=\lim _{n} \operatorname{Tx}_{n} \Omega=$ $\lim _{n} U x_{n}=U x$. The last equality is valid because $U$ is bounded.

Q.E.D.

Theorem II.3 describes completely the Hilbert space $\tilde{\mathscr{H}}$. We notice finally that the algebra $\mathfrak{B}$ of analytic elements of $\mathfrak{M}$ is dense in $\tilde{\mathscr{H}}$. This can be seen as follows: let $x$ be any element of $\tilde{\mathscr{H}}$, then there exists a sequence $\left\{x_{n}\right\}_{n}$ in $\mathfrak{M}$ such that $\lim _{n} x_{n}=x$. As $\mathfrak{M} \Omega \subset \mathscr{D}(T)$, there exists a sequence $\left\{y_{n, l}\right\}_{l}$ in $\mathfrak{B}$ such that

(i) $\lim _{l} y_{n, l} \Omega=x_{n} \Omega$,

(ii) $\lim _{l} T y_{n, l} \Omega=T x_{n} \Omega$.

Let $\varepsilon>0$ be any positive number, choose $n$ such that $\left\|x-x_{n}\right\|_{\sim}<\varepsilon / 2$ and $l$ such that

$$
\left\|x_{n}-y_{n, l}\right\|_{\sim}<\varepsilon / 2
$$

then

$$
\left\|x-y_{n, l}\right\|_{\sim} \leqq\left\|x-x_{n}\right\|_{\sim}+\left\|x_{n}-y_{n, l}\right\|_{\sim}<\varepsilon .
$$

In the following theorem we prove that the scalar product which we introduced, is equivalent with a scalar product widely used in linear response theory (see e.g. [11]).

Theorem II.4. Let $F(y, x)$ for $x, y \in \mathfrak{M}$ be defined $b y$

$$
F(y, x)=\int_{-1}^{0} d t F_{y^{*} x}(i t)
$$


where $F_{y^{*} x}(i t)$ is defined in Definition II.1 then:

(i) $F(y, x)=\int_{0}^{1 / 2} d t\left\{\left(y \Omega, \Delta^{t} x \Omega\right)+\left(x^{*} \Omega, \Delta^{t} y^{*} \Omega\right)\right\}$

$$
=\int_{0}^{1 / 2} d t\left\{\left(y \Omega, \Delta^{t} x \Omega\right)+\left(\Delta^{1 / 2} y \Omega, \Delta^{-t} \Delta^{1 / 2} x \Omega\right)\right\},
$$

(ii) $F(x, y)=(x, y)_{\sim}$.

Proof. Take $F(y, x)$ as above, from a consequence of KMS-state and performing some substitutions we get subsequently

$$
\begin{aligned}
F(y, x) & =\int_{-1 / 2}^{0} d t F_{y^{*} x}(i t)+\int_{-1}^{-1 / 2} d t F_{y^{*} x}(i t) \\
& =\int_{-1 / 2}^{0} d t\left(y \Omega, \Delta^{-t} x \Omega\right)+\int_{-1}^{-1 / 2} d t\left(x^{*} \Omega, \Delta^{1+t} y^{*} \Omega\right) \\
& =\int_{0}^{1 / 2} d t\left(y \Omega, \Delta^{t} x \Omega\right)+\int_{1 / 2}^{1} d t\left(x^{*} \Omega, \Delta^{1-t} y^{*} \Omega\right) \\
& =\int_{0}^{1 / 2} d t\left\{\left(y \Omega, \Delta^{t} x \Omega\right)+\left(x^{*} \Omega, \Delta^{t} y^{*} \Omega\right)\right\} .
\end{aligned}
$$

The second equality of (i) follows from

$$
\begin{aligned}
\left(x^{*} \Omega, \Delta^{t} y^{*} \Omega\right) & =\left(J \Delta^{1 / 2} x \Omega, \Delta^{t} J \Delta^{1 / 2} y \Omega\right) \\
& =\left(J \Delta^{t} J \Delta^{1 / 2} y \Omega, \Delta^{1 / 2} x \Omega\right) \\
& =\left(\Delta^{-t} \Delta^{1 / 2} y \Omega, \Delta^{1 / 2} x \Omega\right) .
\end{aligned}
$$

Now we prove (ii). For any element $x \in \mathfrak{B}$, one easily checks that

$$
\begin{aligned}
\int_{0}^{1_{0} / 2} d t \Delta^{t} x \Omega & =\frac{\Delta^{1 / 2}-1}{\ln \Delta} x \Omega, \\
\int_{0}^{1 / 2} d t \Delta^{-t} x \Omega & =\frac{1-\Delta^{-1 / 2}}{\ln \Delta} x \Omega .
\end{aligned}
$$

Hence for $y \in \mathfrak{M}$ and $x \in \mathfrak{B}$ from (i)

$$
\begin{aligned}
F(y, x) & =\left(y \Omega, \frac{\Delta^{1 / 2}-1}{\ln \Delta} x \Omega\right)+\left(y \Omega, \frac{\Delta-\Delta^{1 / 2}}{\ln \Delta} x \Omega\right) \\
& =\left(y \Omega, \frac{\Delta-1}{\ln \Delta} x \Omega\right)=(T y \Omega, T x \Omega)=(y, x)^{2} .
\end{aligned}
$$

Now we prove the equality for $x$ and $y$ in $\mathfrak{M}$.

As $\mathfrak{B} \Omega$ is dense in $\mathscr{D}\left(\Delta^{1 / 2}\right)$ for any $x \in \mathfrak{M}$ there exists a sequence $\left\{x_{n}\right\}_{n}$ in $\mathfrak{B}$ such that $x_{n} \Omega$ tends to $x \Omega$ and $\Delta^{1 / 2} x_{n} \Omega$ tends to $\Delta^{1 / 2} x \Omega$, hence $x_{n}^{*} \Omega$ tends to $x^{*} \Omega$. We show that $\lim _{n} F\left(y, x_{n}\right)=F(y, x)$. By formula (i)

$$
\begin{aligned}
\lim _{n} F\left(y, x_{n}\right) & =\lim _{n} \int_{0}^{1 / 2} d t\left\{\left(\Delta^{t} y \Omega, x_{n} \Omega\right)+\left(x_{n}^{*} \Omega, \Delta^{t} y^{*} \Omega\right)\right\} \\
& =\int_{0}^{1 / 2} d t\left\{\left(\Delta^{t} y \Omega, x \Omega\right)+\left(x^{*} \Omega, \Delta^{t} y^{*} \Omega\right)\right\} \\
& =F(y, x) .
\end{aligned}
$$

Finally

$$
F(y, x)=\lim _{n} F\left(y, x_{n}\right)=\lim _{n}\left(y, x_{n}\right)_{\sim}=(y, x)_{\sim},
$$

because $T$ is relatively bounded to $\Delta^{1 / 2}$. 
In the following theorem we give another characterization of the scalar product, by establishing a differential equation for it.

Theorem II.5. For all $x, y \in \mathfrak{M}$ we have

$i \frac{d}{d t}\left(x, y_{t}\right)=-\omega\left(\left[x^{*} y_{t}\right]\right)$.

Proof. For $x, y \in \mathfrak{M}$ by definition:

$\left(x, y_{t}\right)_{\sim}=\left(T x \Omega, T y_{t} \Omega\right)$

$$
=\left(T \frac{1}{(1+\Delta)^{1 / 2}}(1+\Delta)^{1 / 2} x \Omega, T \frac{1}{(1+\Delta)^{1 / 2}}(1+\Delta)^{1 / 2} y_{t} \Omega\right) .
$$

Define:

$$
A=\int_{\infty}^{\infty}\left(\frac{1-e^{-\lambda}}{\lambda\left(1+e^{-\lambda}\right)}\right)^{1 / 2} E(d \lambda) .
$$

$A$ is a bounded operator, hence

$$
\begin{aligned}
\left(x, y_{t}\right)_{\sim} & =\left(A(1+\Delta)^{1 / 2} x \Omega, A(1+\Delta)^{1 / 2} y_{t} \Omega\right) \\
& =\left((1+\Delta)^{1 / 2} x \Omega, e^{i t H} A^{2}(1+\Delta)^{1 / 2} y_{t} \Omega\right) .
\end{aligned}
$$

It is clear that the range of $A^{2}$ is contained in the domain of $H$, hence

$$
\begin{aligned}
\frac{1}{i} \frac{d}{d t}(x, y)_{\sim} & =\left((1+\Delta)^{1 / 2} x \Omega,\left(\frac{1-\Delta}{1+\Delta}\right)(1+\Delta)^{1 / 2} y_{t} \Omega\right) \\
& =\left(x \Omega, y_{t} \Omega\right)-\left(\frac{\Delta^{1 / 2}}{(1+\Delta)^{1 / 2}}(1+\Delta)^{1 / 2} x \Omega, \frac{\Delta^{1 / 2}}{(1+\Delta)^{1 / 2}}(1+\Delta)^{1 / 2} y_{t} \Omega\right) \\
& =\left(x \Omega, y_{t} \Omega\right)-\left(\Delta^{1 / 2} x \Omega, \Delta^{1 / 2} y_{t} \Omega\right) \\
& =\omega\left(\left[x^{*}, y_{t}\right]\right) .
\end{aligned}
$$

\section{Operator Representation of Linear Response Theory}

Linearized non-equilibrium statistical mechanics, usually called linear response theory, is studied in terms of the following functions:

- the correlation function $\Psi_{x y}(t)$ : where for $x, y \in \mathfrak{M}, t \in \mathbb{R}$

$$
\Psi_{x y}(t)=\omega\left(\left\{x_{t}, y\right\}_{+}\right),
$$

- the response function $\phi_{x y}(t)$; where for $x, y \in \mathfrak{M}, t \in \mathbb{R}$

$$
\phi_{x y}(t)=\omega\left(\left[x_{t}, y\right]\right),
$$

- the relaxation function $\Phi_{x y}(t)$; where for $x, y \in \mathfrak{M}, t \in \mathbb{R}$

$\Phi_{x y}(t)=\lim _{\varepsilon \rightarrow 0^{+}} i \int_{t}^{\infty} \phi_{x y}\left(t^{\prime}\right) e^{-\varepsilon t^{\prime}} d t^{\prime}$,

if the limit $\varepsilon \rightarrow 0^{+}$exists, 
- the admittance $\chi_{x y}(z)$; where for $x, y \in \mathfrak{M}, \operatorname{Im} z \lessgtr 0$

$$
\chi_{x y}(z)=i \int_{0}^{\infty} d t e^{\mp i z t} \phi_{x y}(t) \text {. }
$$

All these functions not only depend on the time but also depend on the observables $x$ and $y$, and as some properties, in particular the ergodic properties, of the system are reflected in particular properties of these functions valid for all observables, it is natural to look for the basic quantities behind these functions. Especially we associate an operator on the Hilbert space $\tilde{\mathscr{H}}$ with each of these functions. This is what we call the operator representation of linear response theory.

Once done this, the study of linear response theory is reduced to the study of these operators. Moreover all this operators are functions only of the Hamiltonian on $\tilde{\mathscr{H}}$, whose spectrum is, in view of Theorem II.3, unitarily equivalent with the spectrum of the original Hamiltonian $H$ on $\mathscr{H}$. Another advantage of this operator representation is an easy proof of some known and unknown relations between the functions of linear response theory. Maybe, however, the most interesting result is the proof of the existence of the relaxation function and the static admittance and their explicit form.

We constructed in Section II, the Hilbert space $\tilde{\mathscr{H}}$. It was proved that $\tilde{\mathscr{H}}$ is unitarily equivalent with $\mathscr{H}$, and the unitary operator $U$ is defined by

$$
U x=T x \Omega \text { for all } x \in \mathfrak{M} .
$$

Consider the orbit $\left\{x_{t} \mid t \in \mathbb{R}\right\}$ in $\tilde{\mathscr{H}}$ with $x \in \mathfrak{M}$ then

$$
\begin{aligned}
x_{t} & =U^{-1} T x_{t} \Omega=U^{-1} T e^{i t H} x \Omega \\
& =U^{-1} e^{i t H} T x \Omega=U^{-1} e^{i t H} U x, \quad t \in \mathbb{R} .
\end{aligned}
$$

Let $\tilde{H}=U^{-1} H U$ then

$$
x_{t}=e^{i t \tilde{H}} x
$$

hence $\tilde{H}$ is the Hamiltonian on $\tilde{\mathscr{H}}$. The action of the new Hamiltonian is determined by the following theorem.

Theorem III.1. The set $\mathfrak{B}$ is contained in the domain $\mathscr{D}(\tilde{H})$ of $\tilde{H}$ and $\tilde{H} x=H x$ for $x \in \mathfrak{B}$. Moreover $\mathfrak{B}$ is a core for $\tilde{H}$.

Proof. As $\tilde{H}=U^{-1} H U, x \in \mathscr{D}(\tilde{H})$ is equivalent with $U x=T x \Omega \in \mathscr{D}(H)$.

Now let $x \in \mathfrak{B}$, then $x \Omega \in \mathscr{D}(T)$ and as $x \Omega$ belongs to the domain of the operator

$$
\int_{-\infty}^{\infty} \lambda\left(\frac{1-e^{-\lambda}}{\lambda}\right)^{1 / 2} E(d \lambda)
$$

then [13, p. 1199]

$\mathfrak{B} \Omega \subset \mathscr{D}(H T)$.

Analogously $\mathfrak{B} \Omega \subset \mathscr{D}(T H)$, hence for $x \in \mathfrak{B}: H T x \Omega=T H x \Omega$. If we prove that $H x$ belongs to $\mathfrak{B}$ for all $x$ of $\mathfrak{B}$, then

$$
\tilde{H} x=U^{-1} H U x=U^{-1} H T x \Omega=U^{-1} T(H x) \Omega=H x .
$$

Now we prove that $x \in \mathfrak{B}$ implies $H x \in \mathfrak{B}$. 
If $x \in \mathfrak{B}$, then there exists an element $y \in \mathfrak{M}$ and a function $f \in \mathscr{E}$ such that $x=f(H) y$, where $f(\lambda)=\int_{-\infty}^{\infty} f_{1}(s) f_{2}(\lambda-s) d s$ for some continuous functions $f_{1}$ and $f_{2}$ with compact support.

As $\lambda f(\lambda)=\int f_{1}(s)(\lambda-s) f_{2}(\lambda-s) d s+\int s f_{1}(s) f_{2}(\lambda-s) d s$ also $\lambda f(\lambda)$ belongs to $\mathscr{E}$. As $f(\lambda)$ and $\lambda f(\lambda)$ are bounded functions

$$
H f(H)=\int_{-\infty}^{\infty} \lambda f(\lambda) E(d \lambda) \quad[14, \text { p. 358] }
$$

and $H x$ belongs to $\mathfrak{B}$.

Finally $x \in \mathscr{D}(\tilde{H})$ is equivalent with $T x \Omega \in \mathscr{D}(H)$ i.e. $x \Omega \in \mathscr{D}(H T)$. As $\mathfrak{B} \Omega$ is a core for $H T$ there exists a sequence $\left\{x_{n} \Omega\right\}_{n}, x_{n} \in \mathfrak{B}$ such that $x_{n} \Omega$ tends to $x \Omega$ and $H T x_{n} \Omega$ tends to $H T x \Omega$. But as $T$ is $H T$-bounded we have also that $T x_{n} \Omega$ tends to $T x \Omega$. for $\tilde{H}$.

Hence $\left\|x_{n}-x\right\|_{\sim}$ tends to zero and $\left\|\tilde{H} x_{n}-\tilde{H} x\right\|_{\sim}$ tends to zero, i.e. $\mathfrak{B}$ is a core

Theorem III.2. (i) For $x \in \mathscr{D}(H)$ and $y \in \mathfrak{M}$ we have

$(y, \tilde{H} x)_{\sim}=\omega\left(\left[y^{*} x\right]\right)$.

(ii) Let $C(\lambda)$, where $\lambda$ is a real parameter, be given by $C(\lambda)=\lambda \frac{1+e^{-\lambda}}{1-e^{-\lambda}}$, then for all $x \in \mathscr{D}(C(\tilde{H}))$ and $y \in \mathfrak{M}$ :

$(y, C(\tilde{H}) x)_{\sim}=\omega\left(\left\{y^{*}, x\right\}\right)$.

Proof. (i) As $x_{t}=e^{i t \tilde{H}} x$ for all $x \in \mathscr{D}(\tilde{H})$ and $y \in \mathfrak{M}$ by Theorem II.5 one gets $\frac{1}{i} \frac{d}{d t}\left(y, x_{t}\right)_{\sim}=(y, \tilde{H} x)_{\sim}=\omega\left(\left[y^{*}, x_{t}\right]\right)$.

(ii) Remark that $x \in \mathscr{D}(C(\tilde{H}))$ is equivalent with $T x \Omega \in \mathscr{D}(C(H))$. Then

$$
\begin{aligned}
\omega\left(\left\{y^{*}, x\right\}\right) & =(y \Omega, x \Omega)+\left(\Delta^{1 / 2} y \Omega, \Delta^{1 / 2} x \Omega\right) \\
& =\left(T^{-1} T y \Omega, T^{-1} T x \Omega\right)+(A T y \Omega, A T x \Omega)
\end{aligned}
$$

where

$$
A=\int_{-\infty}^{\infty}\left(\frac{e^{-\lambda} \lambda}{1-e^{-\lambda}}\right)^{1 / 2} E(d \lambda) .
$$

Because $T x \Omega \in \mathscr{D}(C(H))=\mathscr{D}\left(A^{2}\right) \cap \mathscr{D}\left(T^{-2}\right)$ for all $x \in \mathscr{D}(C(\tilde{H}))$ and $y \in \mathfrak{M}: \omega\left(\left\{y^{*}, x\right\}\right)=$ $(y, C(\tilde{H}) x)_{\sim}$.

Q.E.D.

In Theorem II. 2 we prove that the response function $\phi_{*}^{*}, .(0)$ for $t=0$ corresponds to a self-adjoint operator $\tilde{H}$ on the Hilbert space $\mathscr{\mathscr { H }}$. Then for $t \neq 0$ it corresponds to the operator $e^{-i t \tilde{H}} \tilde{H}$, such that for each pair of observables $(x, y)$ we have $\phi_{y^{*}, x}(t)=\left(y, e^{-i t \tilde{H}} \tilde{H} x\right)$.

We call the operator $e^{-i t \tilde{H}} \tilde{H}$ the response operator. It is clear now that the study of the response function is reduced to the study of the response operator. As by Theorem II.3 the operators $\tilde{H}$ and $H$ are unitary equivalent we proved, how directly the study of the response function is related to the study of the Hamiltonian $H$. 
Also, we proved that the correlation function $\Psi_{0 *, 0}(t)$ corresponds to the operator $e^{-i t \hat{H}} C(\tilde{H})$, called the correlation operator, such that for each pair of observables $\Psi_{y^{*}, x}(t)=\left(y, e^{-i t \tilde{H}} C(\tilde{H}) x\right)$.

In the following theorem we give the operator representation of the admittance.

Theorem III.3. For $\operatorname{Im} z \neq 0$ and $x, y \in \mathfrak{M}$ :

$\chi_{y, x}(z)=\left(y^{*}, \tilde{H} \tilde{R}(-z) x\right)_{\sim}$

where $\tilde{R}(-z)=(\tilde{H}+z)^{-1}$.

The operator $\tilde{H} \tilde{R}(-z)$ is called the admittance operator.

Proof. By Theorem II.5 for $\operatorname{Im} z \lessgtr 0$

$\chi_{y, x}(z)=-\int_{0}^{\infty} d t e^{\mp i z t} \frac{d}{d t}\left(y_{t}^{*}, x\right)_{\sim}$.

By partial integration:

$$
\begin{aligned}
\chi_{y, x}(z) & =\left(y^{*}, x\right)_{\sim} \mp i z \int_{0}^{\infty} d t\left(y_{t}^{*}, x\right)_{\sim} e^{\mp i z t} \\
& =\left(y^{*},(1-z \tilde{R}(-z)) x\right)_{\sim} \\
& =\left(y^{*}, \tilde{H} \tilde{R}(-z) x\right)_{\sim} .
\end{aligned}
$$

In physics the so-called static admittance, i.e. $\lim _{z \rightarrow 0} \chi_{x, y}(z)$ where the limit is taken in some sense, is widely used. In the following theorem we prove the existence of the static admittance operator as the strong limit of the admittance operator, where the limit is taken in a sector of the complex plane.

Theorem III.4. Let $\tilde{E}_{0}$ be the orthogonal projection on the null space of $\tilde{H}$, then

$$
1-\tilde{E}_{0}=s-\lim _{\substack{z \rightarrow 0^{ \pm} \\ z \in C_{\omega}^{ \pm}}} \tilde{H} \tilde{R}(-z)
$$

where $C_{\omega}^{ \pm}=\left\{z \in \mathbb{C}|\operatorname{Im} z \gtrless 0, \omega \leqq| \arg z \mid \leqq \pi-\omega, 0<\omega<\frac{\pi}{2}\right\}$.

Proof. The proof is based on the proof of Lemma 1.9 of Ref. [15]. As $\tilde{H} \tilde{R}(-z)=$ $1-z \tilde{R}(-z)$, we have to prove that $\lim _{z \rightarrow 0^{ \pm}} z \tilde{R}(-z)=\tilde{E}_{0}$. We give the proof for $\operatorname{Im} z>0$. By the spectral theorem for $\xi \in \tilde{\mathscr{H}}$

$$
z \tilde{R}(-z) \xi=\int_{-\infty}^{\infty} \frac{z}{\lambda+z} E(d \lambda) \xi .
$$

For $z$ tending to zero, the integrand $z / \lambda+z$ tends to the Kronecker $\delta$-function $\delta_{\lambda, 0}$.

The convergence of the integral is dominated since

$$
\left|\frac{z}{\lambda+z}\right| \leqq \frac{1}{\sin \omega} \text { for } z \in C_{\omega}^{+}
$$

and by the Lebesgue dominated convergence theorem

$$
\lim _{\substack{z \rightarrow 0^{+} \\ z \in C_{\omega}^{+}}} z \tilde{R}(-z) \xi=\int_{-\infty}^{\infty} \delta_{\lambda, 0} E(d \lambda)=\tilde{E}_{0} \xi .
$$


Remark now the explicit form of the static admittance, as defined in Theorem III.4 and denoted by $\chi_{x y}(0)$; it is given by: for $x, y \in \mathfrak{M}$ :

$$
\chi_{x, y}(0)=\left(x^{*},\left(1-\tilde{E}_{0}\right) y\right)_{\sim}=\left(T x^{*} \Omega,\left(1-E_{0}\right) T y \Omega\right)
$$

where $E_{0}$ is the orthogonal projection on the null space of the Hamiltonian $H$, hence also

$$
\chi_{x, y}(0)=\left(T x^{*} \Omega, T y \Omega\right)-\left(x^{*} \Omega, E_{0} y \Omega\right) .
$$

We stress here the fact that this quantity is always finite, for each pair of observables $x, y$ of $\mathfrak{M}$.

As the last function in the series we treat the relaxation function.

Theorem III.5. The relaxation function exists for all $x, y$ of $\mathfrak{M}$, and is given by

$$
\begin{aligned}
\Phi_{x, y}(t) & =\left(x^{*}, \lim _{\varepsilon \rightarrow 0^{+}} i e^{-t(\varepsilon+i \tilde{H})} \frac{\tilde{H}}{\varepsilon+i \tilde{H}} y\right)_{\sim} \\
& =\left(x^{*}, e^{-i t \tilde{H}}\left(1-\tilde{E}_{0}\right) y\right)_{\sim} .
\end{aligned}
$$

We call $e^{-i t \tilde{H}}\left(1-\tilde{E}_{0}\right)$ the relaxation operator. In particular $\Phi_{x, y}(0)=\chi_{x y}(0)$.

Proof. By Theorem II.5

$$
\begin{aligned}
\Phi_{x, y}(t) & =-\lim _{\varepsilon \rightarrow 0^{+}} \int_{t}^{\infty} \frac{d}{d t^{\prime}}\left(\left(x_{t^{\prime}}^{*}, y\right)_{\sim}\right) e^{-\varepsilon t^{\prime}} d t^{\prime} \\
& =\lim _{\varepsilon \rightarrow 0^{+}}\left[\left(x_{t}^{*}, y\right)_{L} e^{-\varepsilon t}-\varepsilon\left(x^{*}, \int_{t}^{\infty} e^{\left.\left.-i t \tilde{H}^{-\varepsilon \varepsilon t^{\prime}} y\right)_{\sim}\right]}\right.\right. \\
& =\lim _{\varepsilon \rightarrow 0^{+}}\left(x^{*}, e^{-t(\varepsilon+i \tilde{H})} \frac{i \tilde{H}}{\varepsilon+i \tilde{H}} y\right)_{\sim} .
\end{aligned}
$$

And analogous as in the proof of Theorem III.4 we get

$$
\begin{aligned}
\Phi_{x y}(t) & =\left(x^{*}, e^{-i t \tilde{H}}\left(1-\tilde{E}_{0}\right) y\right)_{\sim} \\
& =\left(T x_{t}^{*} \Omega,\left(1-E_{0}\right) T y \Omega\right) .
\end{aligned}
$$

It follows now from this and Theorem III.4 that

$$
\Phi_{x y}(0)=\chi_{x y}(0) \text {. }
$$

In Theorem III.5, we derived a first relation between two functions namely the relaxation function at $t=0$ and the static admittance. In the following theorems we generalize other relations between the functions to infinite systems.

Theorem III.6. For all $x, y$ of $\mathfrak{M}$ and $t \in \mathbb{R}$, we have

$$
i \frac{d}{d t} \Phi_{x, y}(t)=\phi_{x y}(t)
$$

Proof. By the previous theorem

$$
\Phi_{x y}(t)=\left(x^{*}, e^{-i t \tilde{H}}\left(1-\tilde{E}_{0}\right) y\right)_{\sim}
$$


or

$$
\Phi_{x y}(t)=\left(x_{t}^{*}, y\right)_{\sim}-\left(x, \tilde{E}_{0} y\right)_{\sim}
$$

hence

$$
i \frac{d}{d t} \Phi_{x y}(t)=i \frac{d}{d t}\left(x_{t}^{*}, y\right)_{\sim}=\phi_{x y}(t)
$$

where Theorem II.5 is applied.

Theorem III.7. (Fluctuation-Dissipation Theorems). (i) For all $y$ of $\mathfrak{M}$ and $x \in \mathscr{D}(\tilde{H})$ we have

$$
\left(y, C(\tilde{H}) \frac{1-\tilde{\Delta}}{1+\tilde{\Delta}} x\right)_{2}=(y, \tilde{H} x)_{\sim}
$$

where $\tilde{\Delta}=e^{-\grave{H}}$.

(ii) For all $x, y$ of $\mathfrak{M}$ we have

$$
\phi_{x y}(0)=-i P \int_{-x}^{\infty} d t \Psi_{x y}(t) \frac{2}{e^{\pi t}-e^{-\pi t}}
$$

where $P$ stand for the principal values of the integral.

Proof. (i) is an immediate consequence of the definition of the correlation, and is an operator representation of the well-known fluctuation-dissipation theorem [10].

To prove (ii) use the following formula [16, Lemma 2.3]

$$
i \frac{\Delta-1}{\Delta+1}=P \int_{-\infty}^{\infty} \frac{2}{e^{\pi t}-e^{-\pi t}} \Delta^{-i t} d t
$$

where the integral and the principal value are taken in the strong sense on $\mathscr{H}$.

For any pair $x, y$ of $\mathfrak{M}$ we have

$$
\begin{aligned}
& i\left((1+\Delta)^{1 / 2} y \Omega, \frac{\Delta-1}{\Delta+1}(1+\Delta)^{1 / 2} x \Omega\right) \\
& \quad=P \int_{-\infty}^{x} \frac{2}{e^{\pi t}-e^{-\pi t}}\left((1+\Delta)^{1 / 2} y \Omega, \Delta^{-i t}(1+\Delta)^{1 / 2} x \Omega\right) d t .
\end{aligned}
$$

Furthermore,

$$
\begin{aligned}
&\left((1+\Delta)^{1 / 2} y \Omega, \frac{\Delta-1}{\Delta+1}(1+\Delta)^{1 / 2} x \Omega\right) \\
&=\left(\frac{\Delta^{1 / 2}}{(\Delta+1)^{1 / 2}}(1+\Delta)^{1 / 2} y \Omega, \frac{\Delta^{1 / 2}}{(\Delta+1)^{1 / 2}}(1+\Delta)^{1 / 2} x \Omega\right) \\
&-(y \Omega, x \Omega) \\
&=\left(\Delta^{1 / 2} y \Omega, \Delta^{1 / 2} x \Omega\right)-(y \Omega, x \Omega)=\phi_{y^{*} x}(0)
\end{aligned}
$$


and

$$
\begin{aligned}
\left((1+\Delta)^{1 / 2} y \Omega, \Delta^{i t}(1+\Delta)^{1 / 2} x \Omega\right) \\
\quad=\left(\frac{1+\Delta}{(1+\Delta)^{1 / 2}} y \Omega, \Delta^{i t}(1+\Delta)^{1 / 2} x \Omega\right) \\
=\left(y \Omega, \Delta^{-i t} x \Omega\right)+\left(\Delta^{1 / 2} y \Omega, \Delta^{-i t} \Delta^{1 / 2} x \Omega\right) \\
=\Psi_{y^{*} x}(t)
\end{aligned}
$$

and the theorem follows.

Q.E.D.

As a by product we give a proof of the Bogoliubov inequality for infinite systems. For finite spin systems the proof can be found in Ref. [17]. Here we give a proof of this inequality for KMS-states, in order to point out that this inequality corresponds to Schwartz's inequality applied to the scalar product of $\tilde{\mathscr{H}}$, constructed in Section II.

Theorem III.8. For all $y$ of $\mathfrak{M}$ and elements $x$ such that $x \Omega \in \mathscr{D}(H)$ and $x^{*} \Omega \in \mathscr{D}(H)$, we have

$$
\left|\omega\left(\left[y^{*}, x\right]\right)\right|^{2} \leqq \frac{1}{2} \omega\left(\left\{y, y^{*}\right\}\right) \omega\left(\left[[x, H], x^{*}\right]\right) .
$$

Proof. First we prove that $x \in \mathscr{D}(\tilde{H}): x^{*} \Omega=J \Delta^{1 / 2} x \Omega$, and $J \Delta^{1 / 2} x \Omega \in \mathscr{D}(H)$, as $H J=-J H$ this implies that $x \Omega \in \mathscr{D}\left(H \Delta^{1 / 2}\right) \subset \mathscr{D}(H T)$ i.e. $x \in \mathscr{D}(\tilde{H})$.

From Theorem III. 2

$$
\omega\left(\left[y^{*}, x\right]\right)=(y, \tilde{H} x)_{\sim} .
$$

By Schwartz's inequality

$$
\left|\omega\left(\left[y^{*}, x\right]\right)\right|^{2} \leqq\|y\|_{\sim}^{2}\|\tilde{H} x\|_{\sim}^{2} .
$$

Now

$$
\|y\|_{\sim}^{2}=\|T y \Omega\|^{2} \leqq \frac{1}{2}\left\|(1+\Delta)^{1 / 2} y \Omega\right\|^{2}
$$

where we used the inequality

$$
\frac{1-e^{-\lambda}}{\lambda} \leqq \frac{1}{2}\left(1+e^{-\lambda}\right)
$$

Hence

$$
\|y\|_{\sim}^{2} \leqq \omega\left(\left\{y, y^{*}\right\}\right) .
$$

Also

$$
\begin{aligned}
\|\tilde{H} x\|_{\sim}^{2} & =\|H T x \Omega\|^{2}=\int \lambda\left(1-e^{-\lambda}\right) d\|E(\lambda) x \Omega\|^{2} \\
& =(x \Omega, H x \Omega)-\left(\Delta^{1 / 2} x \Omega, H \Delta^{1 / 2} x \Omega\right)
\end{aligned}
$$

because $x \Omega \in \mathscr{D}(H) \cap \mathscr{D}\left(H \Delta^{1 / 2}\right)$.

Finally $\|\tilde{H} x\|_{\sim}^{2}=(x \Omega, H x \Omega)+\left(H x^{*} \Omega, x^{*} \Omega\right)=\omega\left(\left[[x, H], x^{*}\right]\right)$, where we denote

$$
\omega\left(\left[[x, H], x^{*}\right]\right)=\left(\Omega,\left[[x, H], x^{*}\right] \Omega\right) .
$$

Q.E.D. 


\section{References}

1. Haag. R., Hugenholtz, N.M., Winnink. M.: Commun. math. Phys. 5, 215 (1967)

2. Haake, F.: Statistical treatment of open systems by generalized master equations. Springer Tracts in Modern Physics, Vol. 66. Berlin-Heidelberg-New York: Springer 1973

3. Prigogine, I.: The statistical interpretation of non-equilibrium entropy. In: The Boltzman equation, theory and applications. Cohen,E.G.D., Thirring, W., eds., Berlin-Heidelberg-New York: Springer 1973

4. Bongaarts, P.J.M., Fannes, M., Verbeure, A.: Physica 68, 587 (1973)

5. Davies, E. B.: Markovian master equations; to appear in Comm. Math. Phys.

6. Presutti,E., Scacciatelli,E., Sewell,G.S., Wanderlingh, F.: J. Math. Phys. 13, 1085 (1972)

7. Hepp, K., Lieb, E. H.: Helv. Phys. Acta 46. 573 (1973)

8. Robinson, D.W.: $C^{*}$-algebras and quantum statistical mechanics; Lecture given at the Varenna Summer School on $C^{*}$-algebras (1973)

9. Haag, R., Kaster, D., Trych-Pohlmeyer, E. : Commun. math. Phys. 38, 173 (1974)

10. Kubo, R.: J. Phys. Soc. Japan 12, 570 (1957)

11. Mori, H.: Progr. Theor. Phys. 33, 423 (1965)

12. Takesaki, M.: Tomita's theory of modular Hilbert algebras and its applications; Lecture Notes in Mathematıcs 128. Berlin-Heidelberg-New York: Springer 1970

13. Dunford, N., Schwartz, J. T.: Linear operators, Part II. London: Interscience Publishers 1963

14. Kato, T.: Perturbation theory for linear operators. Berlin-Heidelberg-New York: Springer 1966

15. Balslev, E.: Spectral theory of Schrödinger operators of Many-Body systems. Lecture Notes. University of Leuven, Belgium 1971

16. Van Daele, A.: J. Funct. Ann. 15, 378 (1974)

17. Bogoliubov, N. M.: Dubna reports (unpublished); Physik. Abhandl. Sowjet Union 6, 1; 113-229 (1962)

Ruelle, D.: Statistical mechanics. Lemma 5.5. New York: W. A. Benjamin, Inc. 1969

18. Stonc, M.H.: Linear transformations in Hilbert space and their applications to analysis. Providence. Amm. Math. Soc. Colloq. Publ. Vol. 15 (1932)

19. Verbeure, A., Weder, R. A.: Stability in linear response and clusterıng properties. Commun. math. Phys. 44, 101--105(1975)

Communicated by J. L. Lebowitz

A. Verbeure

Institute for Theoretical Physics

Celestijnenlaan $200 \mathrm{D}$

B-3030 Heverlee, Belgium 
